\title{
A Generalization of the Convex Kakeya Problem*
}

\author{
Hee-Kap Ahn ${ }^{1} \quad$ Sang Won Bae ${ }^{2} \quad$ Otfried Cheong ${ }^{3} \quad$ Joachim Gudmundsson $^{4}$ \\ Takeshi Tokuyama ${ }^{5} \quad$ Antoine Vigneron ${ }^{6}$
}

September 12, 2012

\begin{abstract}
Given a set of line segments in the plane, not necessarily finite, what is a convex region of smallest area that contains a translate of each input segment? This question can be seen as a generalization of Kakeya's problem of finding a convex region of smallest area such that a needle can be rotated through 360 degrees within this region. We show that there is always an optimal region that is a triangle, and we give an optimal $\Theta(n \log n)$-time algorithm to compute such a triangle for a given set of $n$ segments. We also show that, if the goal is to minimize the perimeter of the region instead of its area, then placing the segments with their midpoint at the origin and taking their convex hull results in an optimal solution. Finally, we show that for any compact convex figure $G$, the smallest enclosing disk of $G$ is a smallest-perimeter region containing a translate of every rotated copy of $G$.
\end{abstract}

\section{Introduction}

Let $\mathfrak{F}$ be a family of objects in the plane. A translation cover for $\mathfrak{F}$ is a set $K$ such that any object in $\mathfrak{F}$ is contained in a translate of $K[28$. We are interested in determining a convex translation cover for $\mathfrak{F}$ of smallest possible area or perimeter.

Since the convex hull of a set of objects is the smallest convex figure that contains them, this problem can be reformulated as translating the objects in $\mathfrak{F}$ such that the perimeter or the area of their convex hull is minimized. When $\mathfrak{F}$ consists of $n$ objects, we can fix one object and translate the remaining $n-1$ objects. Therefore we can use a vector in $\mathbb{R}^{2(n-1)}$ to represent the translations of $n-1$ objects. Consider the functions $\mathbb{R}^{2(n-1)} \rightarrow \mathbb{R}$ that take a vector in $\mathbb{R}^{2(n-1)}$ and return the perimeter and the area of the convex hull of the fixed object and the translated copies of the $n-1$ other objects. Ahn and Cheong [1] showed that for the perimeter case, this function is convex. They also showed that for the area case, the function is convex if $n=2$. However, this is no longer true when $n>2$, as the following example shows. Let $s_{1}$ be a vertical segment of length one, and let $s_{2}$ and $s_{3}$ be copies of $s_{1}$ rotated by $60^{\circ}$ and $120^{\circ}$. Then the area of their convex hull is minimized when they form an equilateral triangle, so there are two isolated local minima, as shown in Figure 1 This explains why minimizing the perimeter appears to be a much easier problem than minimizing the area of a translation cover.

As a special case of translation covers, we can consider the situation where the family $\mathfrak{F}$ consists of copies of a given compact convex figure $G$, rotated by all angles in $[0,2 \pi)$. In other words, we are asking for a smallest possible convex set $K$ such that $G$ can be placed in $K$ in every possible orientation. We will call such a translation cover a keyhole for $G$ (since a key can be turned fully in a keyhole, it can certainly be placed in every possible orientation).

A classical keyhole or translation cover problem is the Kakeya needle problem. It asks for a minimum area region in the plane, a so-called Kakeya set, in which a needle of length 1 can be rotated through

\footnotetext{
${ }^{*}$ H.-K.A. was supported by NRF grant 2011-0030044 (SRC-GAIA) funded by the government of Korea. J.G. is the recipient of an Australian Research Council Future Fellowship (project number FT100100755). O.C. was supported in part by NRF grant 2011-0030044 (SRC-GAIA), and in part by NRF grant 2011-0016434, both funded by the government of Korea.

${ }^{1}$ POSTECH, South Korea. Email: heekap@postech.ac.kr.

${ }^{2}$ Kyonggi University, South Korea. Email: swbae@kgu.ac.kr.

${ }^{3}$ KAIST, South Korea. Email: otfried@kaist.edu.

${ }^{4}$ University of Sydney and NICTA, Australia. Email: joachim.gudmundsson@sydney.edu.au.

${ }^{5}$ Tohoku University, Japan. Email: tokuyama@dais.is.tohoku.ac.jp.

${ }^{6}$ KAUST, Saudi Arabia. Email: antoine.vigneron@kaust.edu.sa.
} 

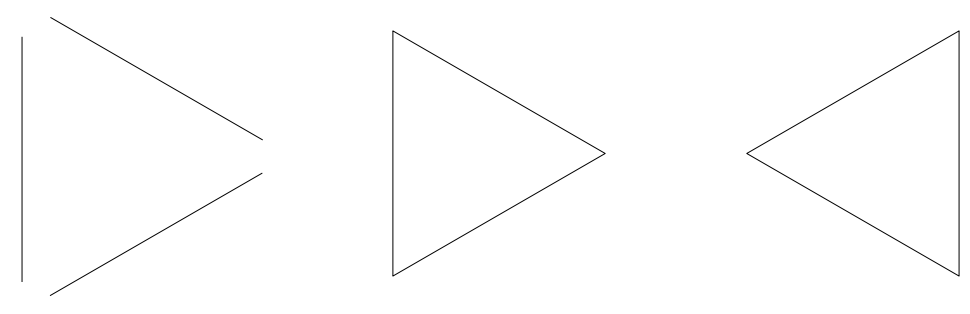

Figure 1: The area function $\omega: \mathbb{R}^{2(n-1)} \rightarrow \mathbb{R}$ of the convex hull of $n \geqslant 3$ segments is not necessarily convex.

$360^{\circ}$ continuously, and return to its initial position. (See Figure 2, This question was first posed, for convex regions, by Soichi Kakeya in 1917 [14. Pàl [17] showed that the solution of Kakeya's problem for convex sets is the equilateral triangle of height one, having area $1 / \sqrt{3}$. With our terminology, he characterized the smallest-area keyhole for a line segment.

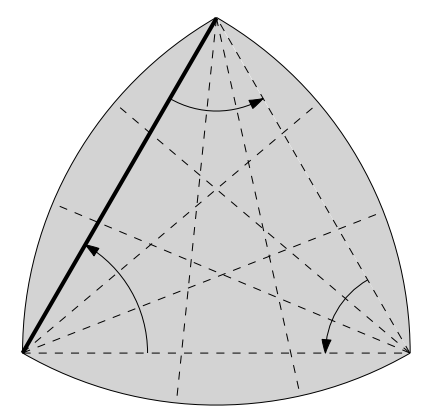

Figure 2: Within a Kakeya set (shaded), a needle can be rotated through $360^{\circ}$.

For the general case, when the Kakeya set is not necessarily convex or even simply connected, the answer was thought to be a deltoid with area $\pi / 8$. However, Besicovitch gave the surprising answer that one could rotate a needle using an arbitrary small area [3, 4.

Besicovitch's solution builds upon two basic observations 24. The first observation is that one can translate any needle to any location using arbitrarily small area. The idea is to slide the needle, rotate it, slide it back and then rotate it back, as illustrated in Fig. 3(a). The area can be made arbitrarily

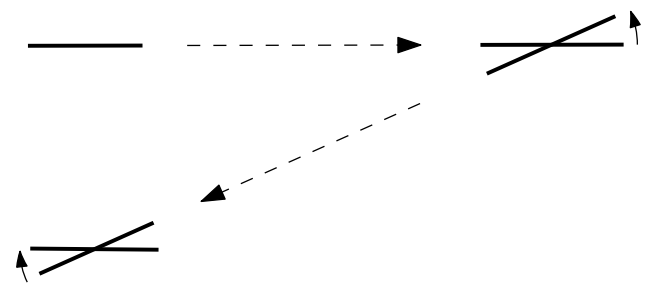

(a)

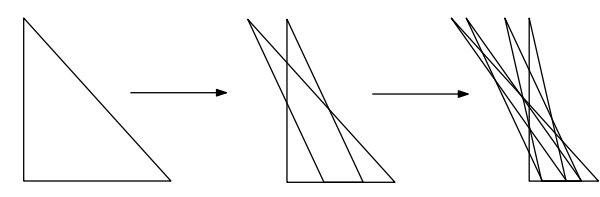

(b)

Figure 3: (a) A needle can be translated to any location using arbitrarily small area. (b) There is an open subset of the plane of arbitrary small area which contain a unit line segment in every direction.

small by sliding the needle over a large distance. The second observation is that one can construct an open subset of the plane of arbitrary small area, which contains a unit line segment in every direction, as illustrated in Fig. 3(b). The original construction by Besicovitch [3, 4] has been simplified by Perron [18], Rademacher [19, Schoenberg [21, 22, Besicovitch [5, 6] and Fisher [12.

Bezdek and Connelly [7] surveyed results on minimum-perimeter and minimum-area translation covers. For the family of closed curves of length at most one, they proved that smallest-perimeter translation covers are exactly the convex sets of constant width $1 / 2$. The corresponding problem for minimizing the area, known as Wetzel's problem, is still open, with upper and lower bounds known [7, 28, For the family of sets of diameter at most one, Bezdek and Connelly [8] proved that the unique minimum-perimeter 
translation cover is the circle of radius $1 / \sqrt{3}$. More precisely, they proved that this circle is the unique smallest-perimeter keyhole for the equilateral triangle of side length one. By Jung's theorem [13, this circle contains any set of diameter one, and so the translation cover result follows.

Recently, Kakeya-type problems have received considerable attention due to their many applications. There are strong connections between Kakeya-type problems and problems in number theory 9], geometric combinatorics [29, arithmetic combinatorics [15, oscillatory integrals, and the analysis of dispersive and wave equations 24 .

In this paper, we first generalize Pál's result [17 in the following way: For any family $\mathfrak{F}$ of line segments in the plane, there is a triangle that is a minimum-area translation cover for $\mathfrak{F}$.

Theorem 1. Let $\mathfrak{F}$ be a set of line segments in the plane, and let $P$ be a convex translation cover for $\mathfrak{F}$. Then there is a translation cover $T$ for $\mathfrak{F}$ which is a triangle, and such that the area of $T$ is less than or equal to the area of $P$.

With this characterization in hand, we can efficiently compute a smallest area translation cover for a given family of $n$ line segments. Our algorithm runs in time $O(n \log n)$, which we prove to be optimal in the algebraic computation tree model. It is based on the problem of finding a smallest-area affine-regular hexagon containing a given centrally symmetric polygon, a problem that is interesting in its own right. As far as we know, except for some trivial cases such as $n$ disks or $n$ axis-aligned squares, previously known algorithms for finding smallest-area translation covers have a running time exponential in $n$, the number of input objects 1, 27.

As observed above, minimizing the perimeter of a translation cover is much easier. Let $\mathfrak{F}$ be a family of centrally symmetric convex figures. We prove that if we translate each figure such that its center of symmetry is the origin, then the convex hull of their union is a smallest-perimeter translation cover for $\mathfrak{F}$.

This immediately implies that a circle with diameter 1 is a smallest-perimeter keyhole for the unitlength segment. For figures $G$ that are not centrally symmetric, this argument no longer works. We generalize the result by Bezdek and Connelly 8 mentioned above and prove the following theorem (Bezdek and Connelly's result is the special case where $G$ is an equilateral triangle):

Theorem 2. Let $G$ be a compact convex set in the plane, and let $\mathcal{G}$ be the family of all the rotated copies of $G$ by angles in $[0,2 \pi)$. Then the smallest enclosing disk of $G$ is a smallest-perimeter translation cover for $\mathcal{G}$.

\section{Preliminaries}

An oval is a compact convex figure in the plane. For an oval $P$, let $w_{P}:[0, \pi] \rightarrow \mathbb{R}$ denote the width function of $P$. The value $w_{P}(\theta)$ is the length of the projection of $P$ on a line with slope $\theta$ (that is, a line that makes angle $\theta$ with the $x$-axis). Let $|P|$ denote the area of $P$.

For two ovals $P$ and $Q$, we write $w_{P} \geqslant w_{Q}$ or $w_{Q} \leqslant w_{P}$ to mean pointwise domination, that is for every $\theta \in[0, \pi)$ we have $w_{P}(\theta) \geqslant w_{Q}(\theta)$. We also write $w_{P}=w_{Q}$ if and only if both $w_{P} \leqslant w_{Q}$ and $w_{Q} \leqslant w_{P}$ hold.

The Minkowski symmetrization of an oval $P$ is the oval $\bar{P}=\frac{1}{2}(P-P)=\left\{\frac{1}{2}(x-y) \mid x, y \in P\right\}$. It is well known and easy to show that $\bar{P}$ is centrally symmetric around the origin, and that $w_{\bar{P}}=w_{P}$.

An oval $D$ is a trigonal disk if there is a centrally symmetric hexagon $A U B V C W$ such that $D$ contains the triangle $A B C$ and is contained in the hexagon $A U B V C W$, as illustrated in Figure 4 (a). Trigonal disks were called "relative Reuleaux triangles" by Ohmann [16] and Chakerian [10, the term "trigonal disk" being due to Fejes Tóth [25] who used it in the context of packings by convex disks. A trigonal disk has three "main" vertices and three arcs connecting these main vertices. For example, the trigonal disk $D$ in Figure 4 (a) consists of three vertices $A, B$, and $C$, and three $\operatorname{arcs}$ connecting them.

Ohmann [16] and Chakerian [10] studied sets with a given fixed width function, and obtained the following result (see for instance Theorem $3^{\prime}$ in [10, for a proof):

Fact 3. Given an oval $P$, there is a trigonal disk $D$ with $|D| \leqslant|P|$ such that $w_{D}=w_{P}$.

\section{Minimum area for a family of segments}

In this section we will prove Theorem 1 The proof contains two parts. First we prove that for every oval $P$ there exists a triangle $T$ with $|T| \leqslant|P|$ and $w_{T} \geqslant w_{P}$ (Theorem प). The second part is to prove that 


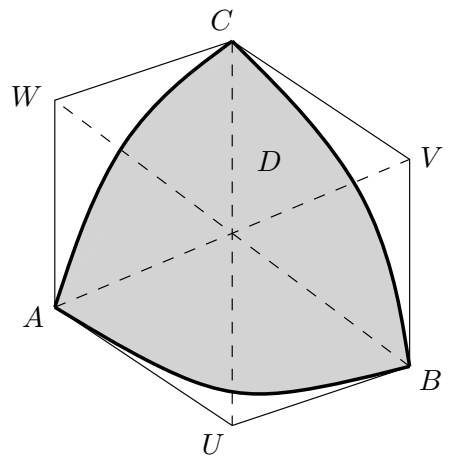

(a)

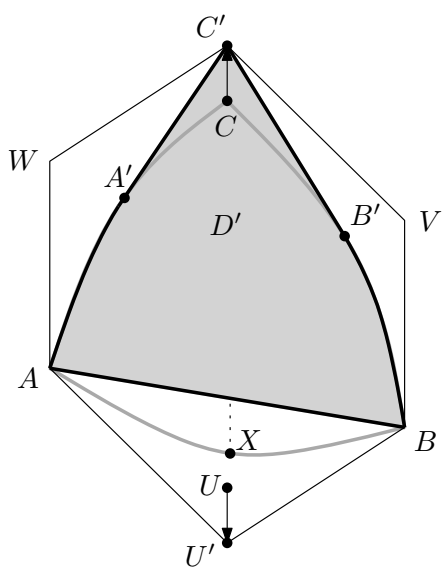

(b)

Figure 4: (a) A trigonal disk $D$ that is contained in the centrally symmetric hexagon $A U B V C W$ and contains the triangle $A B C$. (b) The hexagon $A U^{\prime} B V C^{\prime} W$ is centrally symmetric and contains $D^{\prime}$. Since $D^{\prime}$ contains the triangle $A B C^{\prime}$, it is also a trigonal disk.

for an oval $P$ and a closed segment $s$, if $w_{s} \leqslant w_{P}$ then $P$ contains a translated copy of $s$ (Lemma 5).

Theorem 4. Given an oval $P$, there exists a triangle $T$ with $|T| \leqslant|P|$ and $w_{T} \geqslant w_{P}$.

Proof. Let $\mathfrak{D}$ be the set of trigonal disks $D$ such that we have $|D| \leqslant|P|$ and $w_{D}=w_{P}$. The set $\mathfrak{D}$ is nonempty by Fact 3 . Consider three arcs connecting the main vertices of a trigonal disk in $\mathfrak{D}$. Each arc can be straight, or not. We choose a trigonal disk $D \in \mathfrak{D}$ with a maximum number of straight arcs. We show that $D$ is a triangle.

Let $A U B V C W$ be the hexagon from the definition of the trigonal disk $D$, and assume for a contradiction that $D$ is not a triangle, that is, there is at least one non-straight arc among the three arcs connecting $A, B$, and $C$. See Figure 4(a). Without loss of generality, we assume that the arc connecting $A$ and $B$ is not straight.

Let the sides $A W$ and $B V$ be vertical, with $C$ above the line $A B$. Let $X$ be the point of $D$ below $A B$ with the largest vertical distance $d$ from the line $A B$. Let $C^{\prime}$ be the point vertically above $C$ at distance $d$ from $C$. Let $D^{\prime}$ be the convex hull of the part of $D$ above the line $A B$ and the point $C^{\prime}$. It is not difficult to see that $D^{\prime}$ is also a trigonal disk: Let $U^{\prime}$ be the point vertically below $U$ at distance $d$ from $U$. Then the hexagon $A U^{\prime} B V C^{\prime} W$ is centrally symmetric and contains $D^{\prime}$. Clearly $D^{\prime}$ contains the triangle $A B C^{\prime}$. See Figure $4(\mathrm{~b})$.

We show next that $\left|D^{\prime}\right| \leqslant|D|$. The area of $D^{\prime} \backslash D$ is bounded by the area of the two triangles $A^{\prime} C^{\prime} C$ and $B^{\prime} C^{\prime} C$, where $A^{\prime}$ and $B^{\prime}$ are points on $D$ such that $A^{\prime} C^{\prime}$ and $B^{\prime} C^{\prime}$ are tangent to $D$. This area is equal to $d / 2$ times the horizontal distance between $A^{\prime}$ and $B^{\prime}$. But the horizontal distance between $A^{\prime}$ and $B^{\prime}$ is at most the horizontal distance between $A$ and $B$, so the area of $D^{\prime} \backslash D$ is bounded by the area of the triangle $A X B$, and we have $\left|D^{\prime}\right| \leqslant|D|$.

We also need to argue that $w_{D^{\prime}} \geqslant w_{D}$. Consider a minimal strip containing $D$. If this strip does not touch $D$ from below between $A$ and $B$, then the corresponding strip for $D^{\prime}$ is at least as wide. Otherwise, it touches $D$ from below in a point $Y$ between $A$ and $B$, and touches from above in $C$, as $C$ is the only antipodal point of $D$ for $Y$. A strip with the same direction will be determined either by $A$ and $C^{\prime}$, or by $B$ and $C^{\prime}$, and in both cases its width is not less than the width of the original strip.

Since $w_{D^{\prime}} \geqslant w_{D} \geqslant w_{P}$ and $\left|D^{\prime}\right| \leqslant|D| \leqslant|P|$ the trigonal disk $D^{\prime}$ must be a member of $\mathfrak{D}$. However, $D^{\prime}$ has at least one straight arc more than $D$, contradicting our choice of $D$. It follows that our assumption that $D$ is not a triangle must be false.

This finishes the first part. We need the following lemma, which shows that whether or not an oval $P$ contains a translated copy of a given segment $s$ can be determined by looking at the width functions of $P$ and $s$ alone:

Lemma 5. Let $s$ be a segment in the plane, and let $P$ be an oval such that $w_{s} \leqslant w_{P}$. Then $P$ contains a translated copy of $s$. 
Proof. Without loss of generality, let $s$ be a horizontal segment. Let $p q$ be a horizontal segment of maximal length contained in $P$. Then $P$ has a pair of parallel tangents $\ell_{1}$ and $\ell_{2}$ through $p$ and $q$. By the assumption, the distance between $\ell_{1}$ and $\ell_{2}$ must be large enough to place $s$ in between the two lines. But this implies that the segment $p q$ is at least as long as $s$, and $s$ can be placed on the segment $p q$ in $P$.

To prove Theorem 1, let $P$ be an oval of minimum area that contains a translated copy of every $s \in \mathfrak{F}$. By Theorem 4 there is a triangle $T$ such that $|T| \leqslant|P|$ and $w_{T} \geqslant w_{P}$. Let $s \in \mathfrak{F}$. Since there is a translated copy of $s$ contained in $P$, we must have $w_{s} \leqslant w_{P} \leqslant w_{T}$. By Lemma 5 there is then a translated copy of $s$ contained in $T$.

\section{From triangles to hexagons}

We now turn to the computational problem: Given a family $\mathfrak{F}$ of line segments, find a smallest-area convex set that contains a translated copy of every $s \in \mathfrak{F}$.

By Theorem 1 we can choose the answer to be a triangle. In this section we show that this problem is equivalent to finding a smallest-area affine-regular hexagon enclosing some centrally symmetric convex figure. An affine-regular hexagon is the image of a regular hexagon under a non-singular affine transformation. In this paper, we only consider affine-regular hexagons that are centrally symmetric about the origin, so by abuse of terminology, we will write affine-regular hexagon for an affine-regular hexagon that is centrally symmetric about the origin.

In the next section we will then show how to solve that problem, using the tools of computational geometry.

The basic insight is that for centrally symmetric figures, comparing width-functions is equivalent to inclusion:

Lemma 6. Let $P$ and $Q$ be ovals centrally symmetric about the origin. Then $w_{P} \leqslant w_{Q}$ if and only if $P \subset Q$.

Proof. One direction is trivial, so consider for a contradiction the case where $w_{P} \leqslant w_{Q}$ and $P \not \subset Q$. Then there is a point $p \in P \backslash Q$. Since $Q$ is convex, there is a line $\ell$ that separates $p$ from $Q$. Since $P$ and $Q$ are centrally symmetric, this means that $Q$ is contained in the strip bounded by the lines $\ell$ and $-\ell$, while $P$ contains the points $p$ and $-p$ lying outside this strip. This implies that for the orientation $\theta$ orthogonal to $\ell$ we have $w_{P}(\theta)>w_{Q}(\theta)$, a contradiction.

Recall that $\bar{P}$ denotes the Minkowski symmetrization of an oval $P$.

Lemma 7. Let $T$ be a non-degenerate triangle. Then $\bar{T}$ is an affine-regular hexagon, and $|\bar{T}|=\frac{3}{2}|T|$. Every affine-regular hexagon $H$ can be expressed in this form.

Proof. Since every non-degenerate triangle is the affine image of an equilateral triangle, it suffices to observe this relationship for the equilateral triangle and the regular hexagon.

Since $w_{P}=w_{\bar{P}}, w_{T}=w_{\bar{T}}$, and by Lemmas 6 and 7 , we immediately have

Lemma 8. Given an oval $P$, a triangle $T$ is a smallest-area triangle with $w_{T} \geqslant w_{P}$ if and only if $\bar{T}$ is a smallest-area affine regular hexagon with $\bar{P} \subset \bar{T}$.

This leads us to the following algorithm. In Section [6 we will show that the time bound is tight.

Theorem 9. Let $\mathfrak{F}$ be a set of $n$ line segments in the plane. Then we can find a triangle $T$ in $O(n \log n)$ time which is a minimum-area convex translation cover for $\mathfrak{F}$.

Proof. Given a family $\mathfrak{F}$ of $n$ line segments, place every $s \in \mathfrak{F}$ with its center at the origin. Let $P$ be the convex hull of these translated copies. $P$ can be computed in $O(n \log n)$ time, and is a centrally symmetric convex polygon with at most $2 n$ vertices. We then compute a smallest area affine-regular hexagon $H$ containing $P$. In the next section we will show that this can be done in time $O(n)$. Finally, we return a triangle $T$ with $\bar{T}=H$. The correctness of the algorithm follows from $w_{P}(\theta)=\max _{s \in \mathfrak{F}} w_{s}(\theta)$ and Lemma 8. 


\section{Algorithm for computing the smallest enclosing affine-regular hexagon}

In this section we discuss the following problem: Given a convex polygon $P$, centrally symmetric about the origin, find a smallest-area affine-regular hexagon $H$ such that $P \subset H$.

Let us first sketch a simple quadratic-time algorithm: The affine-regular hexagons centered at the origin are exactly the images of a regular hexagon centered at the origin under a non-singular linear transformation. Instead of minimizing the hexagon, we can fix a regular hexagon $H$ with center at the origin, and find a linear transformation $\sigma$ such that $\sigma P \subset H$ and such that the determinant of $\sigma$ is maximized. The transformation $\sigma$ can be expressed as a $2 \times 2$ matrix with coefficients $a, b, c, d$. The condition $\sigma P \subset H$ can then be written as a set of $6 n$ linear inequalities in the four unknowns $a, b, c, d$. We want to find a feasible solution that maximizes the determinant $a d-b c$, a quadratic expression. This can be done by computing the 4-dimensional polytope of feasible solutions, and considering every facet of this polytope in turn. We triangulate each facet, and solve the maximization problem on each simplex of the triangulation.

In the following, we show that the problem can in fact be solved in linear time.

For a set $S \subset \mathbb{R}^{2}$, let $S^{\circ}=-S$ denote the mirror image with respect to the origin. A strip is the area bounded by a line $\ell$ and its mirror image $\ell^{\circ}$.

An affine-regular hexagon $H$ is the intersection of three strips $\mathfrak{S}_{1}, \mathfrak{S}_{2}$, and $\mathfrak{S}_{3}$, as in Figure 5 , where the sides of $H$ are supported by $\mathfrak{S}_{1}, \mathfrak{S}_{2}, \mathfrak{S}_{3}, \mathfrak{S}_{1}, \mathfrak{S}_{2}$, and $\mathfrak{S}_{3}$ in counter-clockwise order. The intersection

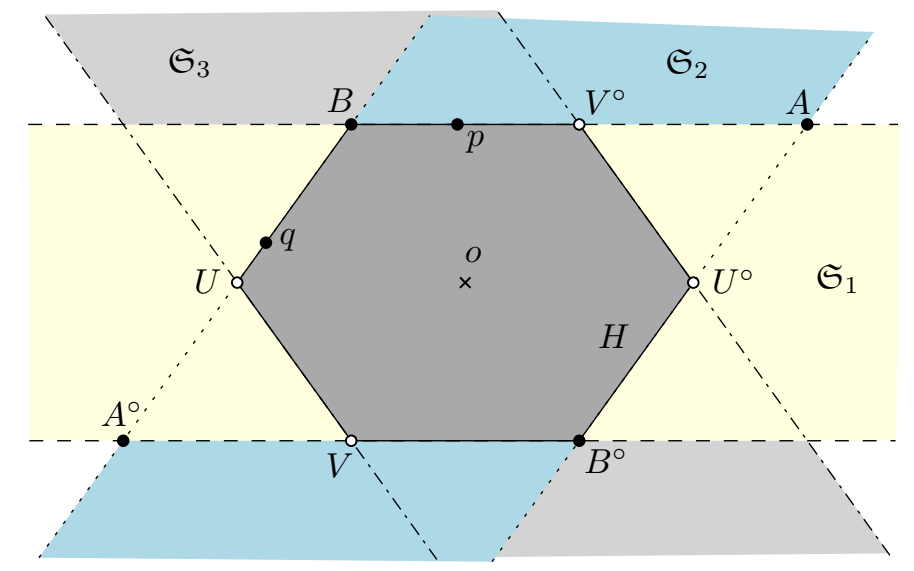

Figure 5: The hexagon $H$ is defined by three strips.

$\mathfrak{S}_{1} \cap \mathfrak{S}_{2}$ is a parallelogram $Q=A B A^{\circ} B^{\circ}$. Since $H$ is affine-regular, the sides supported by $\mathfrak{S}_{3}$ must be parallel to and half the length of $B B^{\circ}$, and so $\mathfrak{S}_{3}$ is uniquely defined by $\mathfrak{S}_{1}$ and $\mathfrak{S}_{2}$ : It supports the sides $U V$ and $U^{\circ} V^{\circ}$ of $H$, where $U$ is the midpoint of $B A^{\circ}$ and $V$ is the midpoint of $A^{\circ} B^{\circ}$. Note that $|H|=3|Q| / 4$.

It is easy to see that if $H$ is a minimum-area affine-regular hexagon containing $P$, then two of the three strips must be touching $P$. Without loss of generality, we can assume these to be strips $\mathfrak{S}_{1}$ and $\mathfrak{S}_{2}$, so there is a vertex $p$ of $P$ on the side $V^{\circ} B$, and a vertex $q \in P$ on the side $B U$.

For convenience of presentation, let us choose a coordinate system where $\mathfrak{S}_{1}$ is horizontal. If we now rotate $\mathfrak{S}_{2}$ counter-clockwise while remaining in contact with $P$, then one side rotates about the point $q$, while the opposite side rotates about $q^{\circ}$, see Figure 6. The triangles $q B B^{\prime}$ and $q A^{\circ} A^{\prime \circ}$ are similar, and since $q$ lies above or on the $x$-axis, we have $\left|q A^{\circ} A^{\prime \circ}\right| \geqslant\left|q B B^{\prime}\right|$. This implies that the area of $Q$ is nonincreasing during this rotation. Since $|H|=3|Q| / 4$, the area of $H$ decreases or remains constant as well.

Furthermore, the point $U$ moves horizontally along the $x$-axis to the right. The point $A^{\circ}$ moves horizontally to the right with at least twice the speed of point $U$. As $V$ is the midpoint of $A^{\circ}$ and $B^{\circ}$, this implies that $V$ moves horizontally to the right with at least the speed of $U$, and so the line $U V$ is rotating counter-clockwise.

It follows that while strip $\mathfrak{S}_{2}$ rotates counter-clockwise, the part of $H$ lying below the $x$-axis and to the left of the line $p p^{\circ}$ is strictly shrinking. It follows that there is a unique orientation of $\mathfrak{S}_{2}$ where the side $U V$ touches $P$, and the area of $Q$ is minimized. 


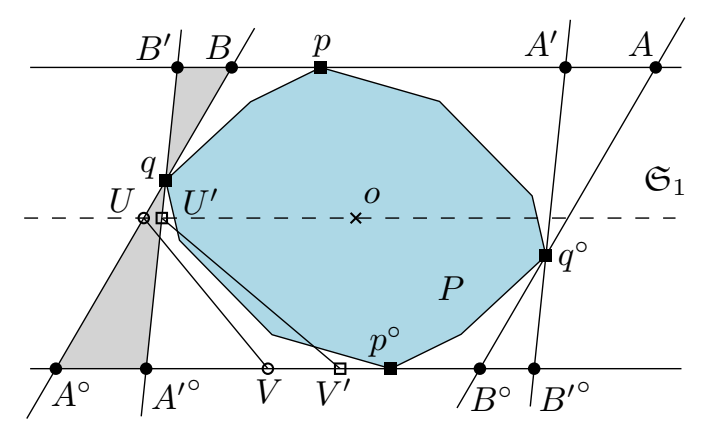

Figure 6: Rotating strip $\mathfrak{S}_{2}$ counter-clockwise.

Let us say that a polygon $S$ is circumscribed to another polygon $R$ if and only if $R \subset S$ and every side of $S$ contains a point of $R$. Then we have shown

Lemma 10. There is a minimum-area affine-regular hexagon $H$ such that $H$ is circumscribed to $P$.

In fact, we have shown that for every $\mathfrak{S}_{1}$ there is a unique $\mathfrak{S}_{2}$ such that $H$ is circumscribed to $P$. We have

Lemma 11. When $\mathfrak{S}_{1}$ rotates counter-clockwise, then the corresponding $\mathfrak{S}_{2}$ also rotates counter-clockwise.

Proof. Consider a configuration where $H$ is circumscribed to $P$, and rotate $\mathfrak{S}_{1}$ slightly around $p$ in counter-clockwise direction, keeping $\mathfrak{S}_{2}$ fixed. Then $B$ and $A^{\circ}$ move downwards along the line $B A^{\circ}$, see Figure 7

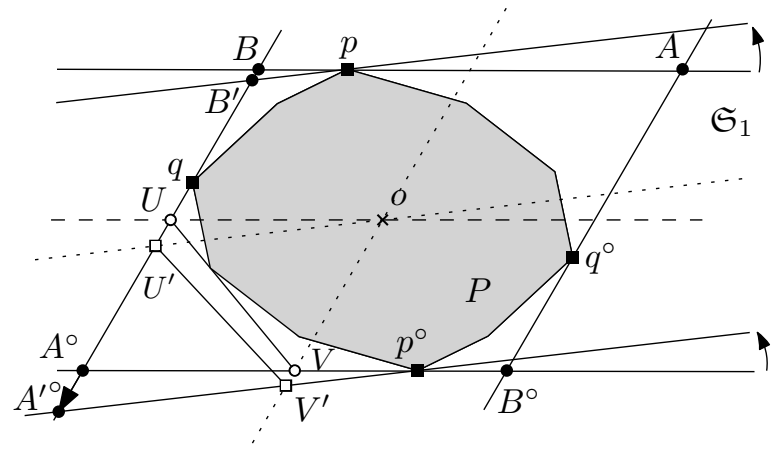

Figure 7: Rotating $\mathfrak{S}_{1}$ counter-clockwise.

The point $V$ moves downwards along the line $o V$, parallel to $B A^{\circ}$. It follows that the new edge $U^{\prime} V^{\prime}$ now lies strictly outside the old hexagon $H$, and so $U^{\prime} V^{\prime}$ cannot possibly touch or intersect $P$. By the arguments above, this implies that strip $\mathfrak{S}_{2}$ now needs to rotate counter-clockwise as well to let $H$ be circumscribed to $P$.

Furthermore, similar to the arguments above, we observe that $A^{\circ}$ moves with speed at least twice the speed of $V$. Since $U$ is the midpoint of $B A^{\circ}$, it moves with at least half the speed of $A^{\circ}$, so $U$ moves with speed at least equal to the speed of $V$. Since $U$ and $V$ move on parallel lines, it follows that the line $U V$ is rotating counter-clockwise during the rotation of $\mathfrak{S}_{1}$.

We can now show that we can in fact choose $H$ such that one of its sides contains an edge of $P$ :

Lemma 12. There exists a minimum-area affine-regular hexagon $H$ containing $P$ such that a side of $H$ contains an edge of $P$. In addition, if no minimum-area affine-regular hexagon containing $P$ shares a vertex with $P$, then each such minimum-area affine-regular hexagon has a side containing a side of $P$.

Proof. By Lemma 10, there exists a minimum-area affine-regular hexagon $H$ such that every side of $H$ contains a point of $P$. If a side of $H$ contains an edge of $P$, then we are done. In the following, we thus assume that every side of $H$ intersects $P$ in a single point. Also, we assume the vertices of $H$ are 


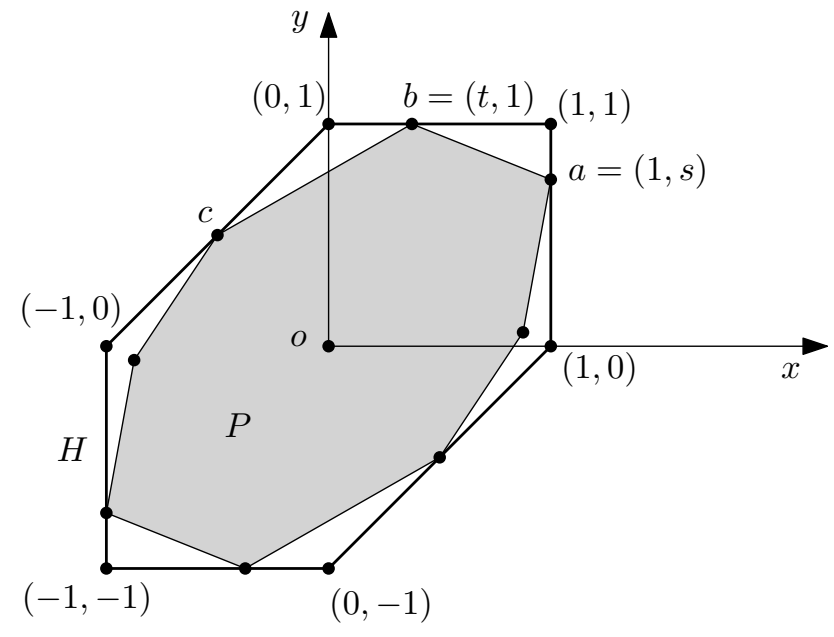

Figure 8: The hexagon $H$, and the convex polygon $P$ (shaded).

$(1,0),(1,1),(0,1)$ and their antipodal points $(-1,0),(-1,-1),(0,-1)$. This can be done by applying a nonsingular linear transformation, see Figure 8.

First, we consider the case where no vertex of $H$ coincides with a vertex of $P$. We claim that in this case, there exists a nonsingular linear transformation $\sigma$ such that $\sigma P \subset H$ and $|\sigma P|>|P|$ hold, implying that the inverse image $\sigma^{-1} H$ of $H$ also contains $P$ and its area $\left|\sigma^{-1} H\right|$ is strictly smaller than $|H|$, a contradiction. We denote by $a, b, c$ the three contact points as in Figure 8 . The point $c$ is a linear combination $c=\alpha a+\beta b$ of $a$ and $b$, so we have $c=(\alpha+\beta t, \alpha s+\beta)$. Since $c$ does not lie in the same quadrant as $a$ and $b$, nor the opposite quadrant, then $\alpha \beta \neq 0$ and $\alpha / \beta<0$. As the point $c$ lies on the line with equation $y=x+1$, we have $t=\frac{\alpha}{\beta} s+\frac{\beta-\alpha-1}{\beta}$. Then the area of the triangle $o a b$ is given by

$$
2|o a b|=1-\frac{\alpha}{\beta} s^{2}-\frac{\beta-\alpha-1}{\beta} s .
$$

Assume we apply a linear transformation $\sigma$ to $P$ such that each point in $a, b, c$ moves along the side of $H$ that currently contains it. Thus, $s$ changes, and $t$ changes in such a way that $c$ remains on the same side of $H$. Then the area of $P$ is proportional to the area $|o a b|$. As we observed that $\alpha / \beta<0$, then the coefficient of $s^{2}$ in Equation (1) is positive, so the area $|o a b|$ cannot be at a local maximum, a contradiction.

Consider now the case where at least one of the contact points $a, b, c$ lies at a vertex of $H$. Since each side of $H$ has a single-point intersection with $P$, two of $a, b, c$ are identical. Using a suitable linear transformation, we can assume $b=c$ in Figure 8 Any linear transformation $\sigma$ that keeps $b=c$ fixed and moves $a$ along the vertical side of $H$ keeps areas unchanged, and so $|\sigma P|=|P|$ and thus $\left|\sigma^{-1} H\right|=|H|$. Hence, there exists a linear transformation $\sigma^{\prime}$ such that $\sigma^{\prime} P \subset H,\left|\sigma^{\prime} P\right|=|P|$, and $\sigma^{\prime} P$ has one more contact point with the sides of $H$.

We can therefore assume that the minimum-area affine-regular hexagon is defined by two strips $\mathfrak{S}_{1}$ and $\mathfrak{S}_{2}$, where $\mathfrak{S}_{1}$ supports an edge of $P$, and $\mathfrak{S}_{2}$ is the unique strip such that the resulting hexagon is circumscribed to $P$. We now give a linear-time algorithm to enumerate these hexagons, over all edges of $P$.

Theorem 13. Given a centrally-symmetric convex 2n-gon $P$, a smallest-area affine-regular hexagon enclosing $P$ can be found in time $O(n)$.

Proof. We use a rotating calipers 26 type algorithm. It maintains an edge $e$ of $P$ defining $\mathfrak{S}_{1}$, a second strip $\mathfrak{S}_{2}$ and the vertex $q$ of $P$ where $\mathfrak{S}_{2}$ touches $P$, and a vertex $r$ of $P$. Let $H=B U V B^{\circ} U^{\circ} V^{\circ}$ be the hexagon defined by $\mathfrak{S}_{1}$ and $\mathfrak{S}_{2}$, as in Figure 5

The algorithm proceeds by rotating $\mathfrak{S}_{2}$ around $q$ as in Figure 6 and maintains the invariant that $P$ has a supporting line in $r$ that is parallel to $U V$.

We initialize $e$ to an arbitrary edge of $P$. Let $e$ be horizontal for ease of presentation, with $P$ below $e$, let $q$ be the left endpoint of $e$, and let $r$ be the leftmost vertex of $P$. In the initial configuration, $\mathfrak{S}_{2}$ is obtained from $\mathfrak{S}_{1}$ by a counter-clockwise rotation around $q$ by an infinitely small amount. 
We then rotate $\mathfrak{S}_{2}$ counter-clockwise, until one of the following events occurs:

- If $r$ no longer supports a tangent to $P$ parallel to $U V$, replace $r$ by the counter-clockwise next vertex of $P$, and continue rotating $\mathfrak{S}_{2}$.

- If $\mathfrak{S}_{2}$ supports an edge of $P$, then replace $q$ by the counter-clockwise next vertex of $P$, and continue rotating $\mathfrak{S}_{2}$.

- If $U V$ touches $r$, then we have found the unique $\mathfrak{S}_{2}$ such that $H$ is circumscribed to $P$. We compute its area and update a running minimum. Then replace $e$ by the counter-clockwise next edge of $P$. As long as $r$ does not support a tangent to $P$ parallel to $U V$, we replace $r$ by the counter-clockwise next vertex of $P$. Then continue rotating $\mathfrak{S}_{2}$.

The algorithm ends when $n$ edges have been considered. Its running time is clearly linear.

\section{Lower bound for computing a translation cover}

In this section, we prove an $\Omega(n \log n)$ lower bound for the problem of computing a minimum-area translation cover for a set of $n$ line segments. We first need the following result on regular $6 n$-gons (see Figure 9(a).):

Lemma 14. Let $R$ denote a regular $6 n$-gon centered at the origin, for some integer $n \geqslant 1$. Then any minimum-area affine-regular hexagon enclosing $R$ is a regular hexagon such that every edge of this hexagon contains an edge of $R$.

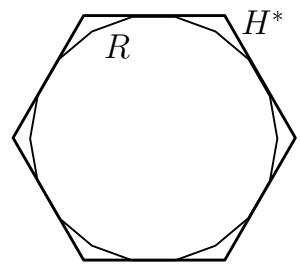

(a)

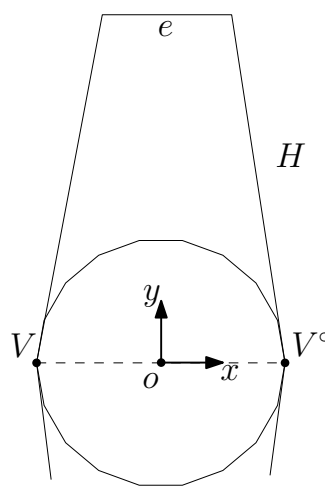

(b)

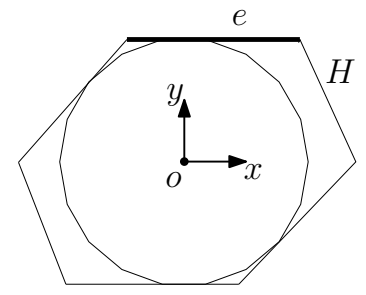

(c)

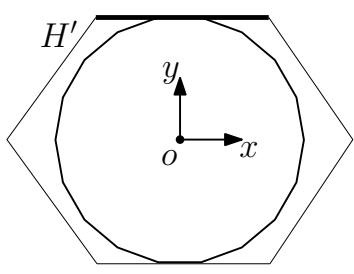

(d)

Figure 9: Proof of Lemma 14, (a) An optimal enclosing hexagon $H^{*}$ and the regular 18-gon $R$. (b) When $H$ and $R$ share two vertices, the area of $H$ is larger than the area of $H^{*}$. (c) An affine-regular enclosing hexagon $H$. (d) The hexagon $H^{\prime}$.

Proof. The statement is trivial for $n=1$, so assume $n \geqslant 2$. Let $H^{*}$ denote a regular hexagon enclosing $R$, and such that each side of $H^{*}$ contains a side of $R$. Let $H$ denote another smallest affine-regular hexagon enclosing $R$. We will argue that $H$ is also a regular hexagon whose sides contain sides of $R$.

We first rule out the case where $H$ shares a vertex with $R$. For sake of contradiction, assume that $H$ shares two opposite vertices $V$ and $V^{\circ}$ of $R$. Without loss of generality, we assume that $V, V^{\circ}$ are on the $x$-axis. The edges $e, e^{\circ}$ of $H$ that are not adjacent to $V, V^{\circ}$ are parallel to $V V^{\circ}$ and have half the length of $\overline{V V^{\circ}}$. In addition, the edges of $H$ that are adjacent to $V$ and $V^{\circ}$ make an angle at most $\pi / 12$ with the $y$-axis. (See Figure 9(b).) Then a direct calculation shows that $|H|>\left|H^{*}\right|$, a contradiction.

Thus, by Lemma 12, we know that an edge $e$ of $H$ contains an edge of $R$. Without loss of generality we assume that this edge is parallel to the $x$-axis. (See Figure 9(c).) For sake of contradiction, assume that $e$ is not symmetric with respect to the $y$-axis. Consider the hexagon $H^{\prime}$ that is obtained from $H$ by a horizontal shear transformation that moves $e$ and the opposite edge parallel to the $x$-axis, until they are centered at the $y$-axis. Then $H^{\prime}$ (see Figure 9(d)) is an affine-regular hexagon containing $R$ that is symmetric with respect to the $y$-axis and that only touches $R$ along its top and bottom edges. This implies that $H^{\prime}$ strictly contains a regular hexagon $H^{*}$ enclosing $R$, and hence $|H|=\left|H^{\prime}\right|>\left|H^{*}\right|$, a contradiction. 
Therefore, $e$ is symmetric with respect to the $y$-axis, and thus $H$ is symmetric with respect to the $y$-axis. Only one such affine-regular hexagon is circumscribed to $R$, so $H=H^{*}$.

We are now able to prove our lower bound.

Theorem 15. In the algebraic computation tree model, and in the worst case, it takes $\Omega(n \log n)$ time to compute a minimum-area translation cover for a family $\mathfrak{F}$ of $n$ line segments in the plane.

Proof. For an interval $I \subset \mathbb{R}$, we denote by $C_{I}$ the arc of the unit circle corresponding with polar angles in the interval $I$, that is $C_{I}=\{(\cos \theta, \sin \theta) \mid \theta \in I\}$. As $C_{I}$ is the intersection of a circle and a cone, a node of an algebraic computation tree can decide whether a point lies in $C_{I}$.

We use a reduction from the following problem. The input is a set of points $p_{1}, \ldots, p_{n} \in C_{[0, \pi / 3)}$. The goal is to decide whether there exists an integer $0 \leqslant k<n$ such that $C_{(k \pi / 3 n,(k+1) \pi / 3 n)}$ is empty, that is, this arc does not contain any point $p_{i}$. It follows from Ben-Or's bound [2] that any algebraic computation tree that decides this problem has depth $\Omega(n \log n)$. (The set of negative instances has at least $n$ ! connected component: To each permutation $\sigma$ of $1, \ldots, n$, we associate a negative instance where each $p_{i}$ lies in the $\sigma_{i}$ 's arc. In order to move continuously from one of these configuration to another, we must have a crossing $p_{i}=p_{j}$, which implies that one interval is empty by the pigeonhole principle, and thus the instance is positive.)

Our construction is as follows. Consider the (fixed) regular $6 n$-gon $R$, whose vertices are $r_{k}=$ $(\cos (k \pi / 3 n), \sin (k \pi / 3 n))$ for $k=1, \ldots, 6 n$. Let $P$ denote the convex $12 n$-gon whose vertices are the vertices of $R$ and all the rotated copies of the points $p_{1}, \ldots, p_{n}$ by angles $0, \pi / 3, \ldots, 5 \pi / 3$ around the origin.

If there is an integer $k=0, \ldots, n-1$ such that $C_{(k \pi / 3 n,(k+1) \pi / 3 n)}$ is empty, then by Lemma 12 , the regular hexagon containing $R$ whose edges contain the edge $r_{k} r_{k+1}$ and its rotated copies by angles $0, \pi / 3, \ldots, 5 \pi / 3$ is a minimum area affine-regular hexagon containing $P$.

If on the other hand, for every integer $k \in\{0, \ldots, n-1\}$ the $\operatorname{arc} C_{(k \pi / 3 n,(k+1) \pi / 3 n)}$ is nonempty, then by Lemma 12, any minimum-area affine hexagon containing $R$ is a regular hexagon whose edges contain edges of $R$, and thus it cannot contain $P$.

So we have proved that, when some arc $C_{(k \pi / 3 n,(k+1) \pi / 3 n)}$ is empty, then a minimum-area hexagon containing $P$ has area $\left|H^{*}\right|$, where $H^{*}$ is a minimum-area hexagon containing $R$. Otherwise, if all these arcs are non-empty, then the minimum area is larger than $\left|H^{*}\right|$.

Thus, if we could compute in $o(n \log n)$ time a minimum-area convex translation cover for the diagonals of $P$, then by Lemma 8 we would also get in $o(n \log n)$ time the area of a smallest enclosing affine-regular hexagon containing $P$, and then we would be able to decide in $o(n \log n)$ time whether there exists an empty arc $C_{(k \pi / 3 n,(k+1) \pi / 3 n)}$, a contradiction.

\section{Minimizing the perimeter}

If we wish to minimize the perimeter instead of the area, the problem becomes much easier: it suffices to translate all segments so that their midpoints are at the origin, and take the convex hull of the translated segments. This follows from the following more general result.

Theorem 16. Let $\mathcal{C}$ be a family of centrally symmetric convex figures. Under translations, the perimeter of the convex hull of their union is minimized when the centers coincide.

Proof. By the Cauchy-Crofton formula [1], the perimeter is the integral of the width of the projection over all directions. We argue that the width is minimized when the centers coincide, for all directions simultaneously, implying the claim.

Assume the objects are placed with their center at the origin. Let $p$ be a leftmost point of the convex hull. It belongs to one of the objects $C \in \mathcal{C}$. By symmetry, the mirror image of $p$ is then a rightmost point of the convex hull. But this implies that the horizontal width of the convex hull is equal to the width of $C$, and therefore as small as possible.

When the figures are not symmetric, our proof of Theorem 16 breaks down. However, we are able to solve the problem for a family consisting of all the rotated copies of a given oval. (Remember that an oval is a compact convex set.) The following theorem was already stated in the introduction. 
Theorem 2, Let $G$ be an oval, and let $\mathcal{G}$ be the family of all the rotated copies of $G$ by angles in $[0,2 \pi)$. Then the smallest enclosing disk of $G$ is a smallest-perimeter translation cover for $\mathcal{G}$.

Proof. We observe first that, if $G$ is a segment, then by Theorem 16. the smallest enclosing disk of $G$ is a smallest-perimeter translation cover for $\mathcal{G}$.

Consider next the case where $G$ is an acute triangle. Choose a coordinate system with origin at the center of the circumcircle of $G$, and such that the circumcircle has radius one. We wish to prove that any translation cover for $\mathcal{G}$ must have perimeter at least $2 \pi$, implying that the circumcircle is optimal.

We borrow an idea of Bezdek and Connelly [8]. Let $v_{1}, v_{2}, v_{3}$ be the three vertices of $G$. By our assumptions, the origin lies in the interior of their convex hull, and the three vectors have length one. The origin can be expressed as a convex combination $0=\sum_{i=1}^{3} \alpha_{i} v_{i}$ with $\alpha_{i} \geqslant 0$ and $\sum_{i=1}^{3} \alpha_{i}=1$. Let $\delta_{i}$, for $i=1,2,3$, be the angle formed by $v_{i}$ and the positive $x$-axis.

Let $K$ be a translation cover for $\mathcal{G}$ and let $h$ be the support function 20 of $K$. That is, $h(u)=$ $\sup \{\langle x, u\rangle \mid x \in K\}$ for any unit vector $u$. We denote by $u_{\theta}=(\cos \theta, \sin \theta)$ the unit vector making angle $\theta$ with the positive $x$-axis, so that $v_{i}=u_{\delta_{i}}$.

The length $\lambda$ of the perimeter of $K$ is equal to the integral over the support function 23

$$
\lambda=\int_{0}^{2 \pi} h\left(u_{\theta}\right) d \theta .
$$

Since $\theta \mapsto h\left(u_{\theta}\right)$ is a periodic function with period $2 \pi$, we have

$$
\lambda=\int_{0}^{2 \pi} h\left(u_{\theta}\right) d \theta=\int_{\delta_{i}}^{2 \pi+\delta_{i}} h\left(u_{\theta}\right) d \theta=\int_{0}^{2 \pi} h\left(u_{\theta+\delta_{i}}\right) d \theta .
$$

It follows that

$$
\lambda=\sum_{i=1}^{3} \alpha_{i} \lambda=\sum_{i=1}^{3} \alpha_{i} \int_{0}^{2 \pi} h\left(u_{\theta+\delta_{i}}\right) d \theta=\int_{0}^{2 \pi}\left(\sum_{i=1}^{3} \alpha_{i} h\left(u_{\theta+\delta_{i}}\right)\right) d \theta .
$$

Consider now a fixed orientation $\theta$. The translation cover $K$ must contain a rotated copy $G(\theta)$ of $G$ such that, for some translation vector $c(\theta)$, the vertices of $G(\theta)$ are the points $v_{i}(\theta)=c(\theta)+u_{\theta+\delta_{i}}$ for $i=1,2,3$.

Since $v_{i}(\theta)$ lies in $K$, the value of the support function $h\left(u_{\theta+\delta_{i}}\right)$ is lower bounded by

$$
h\left(u_{\theta+\delta_{i}}\right) \geqslant\left\langle v_{i}(\theta), u_{\theta+\delta_{i}}\right\rangle=\left\langle c(\theta)+u_{\theta+\delta_{i}}, u_{\theta+\delta_{i}}\right\rangle=\left\langle c(\theta), u_{\theta+\delta_{i}}\right\rangle+1
$$

and thus

$$
\sum_{i=1}^{3} \alpha_{i} h\left(u_{\theta+\delta_{i}}\right) \geqslant 1+\left\langle c(\theta), \sum_{i=1}^{3} \alpha_{i} u_{\theta+\delta_{i}}\right\rangle=1+\langle c(\theta), 0\rangle=1 .
$$

Plugging this into Eq. (2) gives $\lambda \geqslant 2 \pi$.

Consider finally the general case where $G$ is an arbitrary compact convex figure, and let $D$ be the smallest enclosing disk of $G$. Either $D$ touches $G$ in two points that form a diameter of $D$, or $D$ touches $G$ in three points that form an acute triangle. In both cases, our previous results imply that $D$ is a smallest-perimeter translation cover for either the segment or the triangle, and therefore for $G$.

The minimum enclosing circle is not always the unique minimum-perimeter keyhole: For instance, when $G$ is a unit line segment, then any set of constant width is a solution. In the theorem below, we show that when $G$ is an acute triangle, then its circumcircle is the unique solution. This generalizes directly to any figure $G$ that touches its circumcircle at 3 points.

Theorem 17. If $G$ is an acute triangle, then its smallest enclosing disk is the unique smallest-perimeter translation cover for the family of all rotated copies of $G$.

Proof. We use the same notations as in the proof of Theorem 2 $K$ is a smallest-perimeter translation cover for $\mathcal{G}$. For any $\theta$, it contains a copy $G(\theta)$ of $G$ rotated by angle $\theta$. The vertices of $G(\theta)$ are the points $v_{i}(\theta)=c(\theta)+u_{\theta+\delta_{i}}$, for $i=1,2,3$.

We will prove that all the triangles $G(\theta)$ have the same circumcircle. Our strategy is to show that the function $\theta \mapsto c(\theta)$ is differentiable and its derivative is 0 . Without loss of generality, we only prove that $c^{\prime}(0)=0$, and we assume that $c(0)=0$. 
For sake of contradiction, assume that $c$ is not differentiable at 0 , or it is differentiable at 0 and its derivative is nonzero. This means that we do not have $\lim _{\theta \rightarrow 0} c(\theta) / \theta=0$. Hence, there exists an $\varepsilon>0$ such that for any integer $n$, there exists $\theta_{n} \in(-1 / n, 0) \cup(0,1 / n)$ with $\left\|c\left(\theta_{n}\right) / \theta_{n}\right\|>\varepsilon$. This implies $c\left(\theta_{n}\right) \neq 0$, and so $c\left(\theta_{n}\right) /\left\|c\left(\theta_{n}\right)\right\|$ is a sequence of unit vectors. Since the set of unit vectors is compact, there is a subsequence $\left(\theta_{n_{k}}\right)$ such that $c\left(\theta_{n_{k}}\right) /\left\|c\left(\theta_{n_{k}}\right)\right\|$ converges to a unit vector $c_{0}$. We denote this subsequence again as $\left(\theta_{n}\right)$.

Since $u_{\delta_{1}}, u_{\delta_{2}}, u_{\delta_{3}}$ span $\mathbb{R}^{2}$, there exists $i \in\{1,2,3\}$ such that $\left\langle c_{0}, u_{\delta_{i}}\right\rangle>0$. So

$$
\lim _{n \rightarrow \infty} \frac{1}{\left\|c\left(\theta_{n}\right)\right\|}\left\langle c\left(\theta_{n}\right), u_{\delta_{i}}\right\rangle=\left\langle c_{0}, u_{\delta_{i}}\right\rangle>0
$$

As $\left\|c\left(\theta_{n}\right)\right\|>\varepsilon\left\|\theta_{n}\right\|$ for all $n$, this implies that for $n$ large enough,

$$
\left\langle c\left(\theta_{n}\right), u_{\delta_{i}}\right\rangle>\frac{\varepsilon\left\|\theta_{n}\right\|}{2}\left\langle c_{0}, u_{\delta_{i}}\right\rangle,
$$

hence

$$
\begin{aligned}
\left\langle v_{i}\left(\theta_{n}\right), u_{\delta_{i}}\right\rangle & =\left\langle c\left(\theta_{n}\right)+u_{\theta_{n}+\delta_{i}}, u_{\delta_{i}}\right\rangle \\
& >\frac{\varepsilon\left\|\theta_{n}\right\|}{2}\left\langle c_{0}, u_{\delta_{i}}\right\rangle+\cos \left(\theta_{n}\right) \\
& =1+\frac{\varepsilon\left\|\theta_{n}\right\|}{2}\left\langle c_{0}, u_{\delta_{i}}\right\rangle-\frac{\theta_{n}^{2}}{2}+o\left(\theta_{n}^{3}\right) .
\end{aligned}
$$

Thus, for large enough $n$, we have $\left\langle v_{i}\left(\theta_{n}\right), u_{\delta_{i}}\right\rangle>1$. Since $v_{i}\left(\theta_{n}\right) \in K$ for all $n$, this implies $h\left(u_{\delta_{i}}\right)>1$. But since $c(0)=0$, this means $h\left(u_{\delta_{i}}\right)>1+\left\langle c(0), u_{\delta_{i}}\right\rangle$, and so Inequality (3) in the proof of Theorem 2 is not tight. Since the support function $h$ is continuous [20], this implies that $\lambda>2 \pi$, a contradiction.

\section{Conclusions}

In practice, it is an important question to find the smallest convex container into which a family of ovals can be translated. For the perimeter, this is answered by the previous lemma for centrally symmetric ovals. For general ovals, it is still not difficult, as the perimeter of the convex hull is a convex function under translations [1. This means that the problem can be solved in practice by numerical methods.

For minimizing the area, the problem appears much harder, as there can be multiple local minima. The following lemma solves a very special case.

Lemma 18. Let $\mathcal{R}$ be a family of axis-parallel rectangles. The area of their convex hull is minimized if their bottom left corners coincide (or equivalently if their centers coincide).

Proof. Let $C$ be the convex hull of some placement of the rectangles. For any $x$, let $\ell(x)$ be the length of the intersection of the vertical line at coordinate $x$ with $C$. The function $x \mapsto \ell(x)$ is concave (by the Brunn-Minkowski theorem in two dimensions). For any $z \geqslant 0$, we define $w(z)$ to be the length of the interval of all $x$ where $\ell(x) \geqslant z$.

We observe that the area of $C$ is equal to $\int \ell(x) d x$, which is again equal to $\int_{0}^{\infty} w(z) d z$. We will now argue that $w(z)$ is minimized for every $z$ when the bottom left corners of the rectangles coincide, implying the claim.

To see this, consider the placement with coinciding bottom left corners at the origin, and the line $y=z$. It intersects the convex hull at $x=0$ and at some convex hull edge defined by two rectangles $R_{1}$ and $R_{2} . w(z)$ is equal to the length of this intersection. It remains to observe that for any placement of $R_{1}$ and $R_{2}$, the convex hull of these two rectangle already enforces this value of $w(z)$.

\section{Acknowledgments}

We thank Helmut Alt, Tetsuo Asano, Jinhee Chun, Dong Hyun Kim, Mira Lee, Yoshio Okamoto, János Pach, Günter Rote, and Micha Sharir for helpful discussions. 


\section{References}

[1] H.-K. Ahn and O. Cheong. Aligning two convex figures to minimize area or perimeter. Algorithmica, 62:464-479, 2012.

[2] M. Ben-Or. Lower bounds for algebraic computation trees. In Proceedings of the 15th Annual ACM Symposium on Theory of Computing, pages 80-86, 1983.

[3] A. S. Besicovitch. Sur deux questions de l'intégrabilité. Journal de la Société des Math. et de Phys., II, 1920.

[4] A. S. Besicovitch. On Kakeya's problem and a similar one. Math. Zeitschrift, 27:312-320, 1928.

[5] A. S. Besicovitch. The Kakeya problem. American Math. Monthly, 70:697-706, 1963.

[6] A. S. Besicovitch. On fundamental geometric properties of plane line sets. Journal of the London Math. Society, 39:441-448, 1964.

[7] K. Bezdek and R. Connelly. Covering curves by translates of a convex set. American Math. Monthly, 96:789-806, 1989.

[8] K. Bezdek and R. Connelly. The minimum mean width translation cover for sets of diameter one. Beiträge zur Algebra und Geometrie, 39:473-479, 1998.

[9] J. Bourgain. Harmonic analysis and combinatorics: How much may they contribute to each other? In V. I. Arnold, M. Atiyah, P. Lax, and B. Mazur, editors, Mathematics: Frontiers and Perspectives, pages 13-32. American Math. Sociaty, 2000.

[10] G. D. Chakerian. Sets of constant width. Pacific J. of Math., 19:13-21, 1966.

[11] M. do Carmo. Differential Geometry of Curves and Surfaces. Prentice-Hall, 1976.

[12] B. Fisher. On a problem of Besicovitch. American Math. Monthly, 80(7):785-787, 1973.

[13] H. Jung. Über die kleinste Kugel, die eine räumliche Figur einschliesst. J. Reine Angew. Math., 123:241-257, 1901.

[14] S. Kakeya. Some problems on maximum and minimum regarding ovals. Tohoku Science Reports, 6:71-88, 1917.

[15] I. Laba. From harmonic analysis to arithmetic combinatorics. Bulletin (New Series) of the AMS, 45(1):77-115, 2008.

[16] D. Ohmann. Extremalprobleme für konvexe Bereiche der euklidischen Ebene. Math. Zeitschrift, 55:346-352, 1952.

[17] G. Pál. Ein Minimumproblem für Ovale. Math. Ann., 83:311-319, 1921.

[18] O. Perron. Über einen Satz von Besicovitch. Math. Zeitschrift, 28:383-386, 1928.

[19] H. A. Rademacher. On a theorem from Besicovitch. In G. Szego, editor, Studies in Mathematical Analysis and Related Topics: Essays in Honor of George Pòlya, pages 294-296. Stanford University Press, 1962.

[20] R. Schneider. Convex Bodies: The Brunn-Minkowski Theory. Cambridge University Press, 1993.

[21] I. J. Schoenberg. On certain minima related to the Besicovitch-Kakeya problem. Mathematika, $4(27): 145-148,1962$.

[22] I. J. Schoenberg. On the Besicovitch-Perron solution of the Kakeya problem. In G. Szego, editor, Studies in Mathematical Analysis and Related Topics: Essays in Honor of George Pòlya, pages 359-363. Stanford University Press, 1962. 
[23] G. Strang. Maximum area with Minkowski measures of perimeter. Proc. of the Royal Society of Edinburgh, 138A:189-199, 2008.

[24] T. Tao. From rotating needles to stability of waves: Emerging connections between combinatorics, analysis and PDE. Notices of the AMS, 48(3):297-303, 2001.

[25] L. F. Tóth. On the densest packing of convex disks. Mathematika, 30:1-3, 1983.

[26] G. Toussaint. Solving geometric problems with the rotating calipers. In Proceedings of IEEE MELECON, pages 1-4, 1983.

[27] A. Vigneron. Geometric optimization and sums of algebraic functions. In Proceedings of the 21st ACM-SIAM Symposium on Discrete Algorithms, pages 906-917, 2010.

[28] J. E. Wetzel. Sectorial covers for curves of constant length. Canad. Math. Bull., 16:367-375, 1973.

[29] T. Wolff. Recent work connected with the Kakeya problem. In H. Rossi, editor, Prospects in Mathematics. American Math. Sociaty, 1999. 